\section{Urologia Internationalis}

associata con

ACTA

UROLOGICA ITALICA

Organo Ufficiale della

Società Italiana di Urologia

\title{
Verso il Congresso del 2000
}

Il $73^{\circ}$ Congresso Nazionale della Società Italiana di Urologia si svolgerà a Bologna dal 28 al 31 maggio prossimo. L'inaugurazione sarà domenica 28 maggio alle ore 18.00 nell'Aula magna di Santa Lucia, i lavori congressuali nei giorni seguenti, nelle 5 aule dell'Ente Fiera.

Bologna, per la sua posizione, per la sua disponibilità alberghiera e per le diverse strutture congressuali appare essere una città adatta ad accogliere i congressisti che, nella nostra specialità sono sempre numerosi e partecipi. Quest'anno Bologna 2000 è anche Città Europea della Cultura e quindi risulta essere la sede più adatta ad una accoglienza culturale che il nostro Congresso non può non avere.

Questo $73^{\circ}$ Congresso può essere definito di transizione perché entrerà in vigore il nuovo statuto votato con non poche difficoltà a Roma e con molti, forse troppi, emendamenti al testo proposto dal CD uscente.

La nostra Società cioè deve transitare dalla gestione un po' autoritaria, anche se efficace, dei nostri Maestri, ad una più articolata e partecipe di tutti i Soci. A questo cambiamento il $\mathrm{CD}$ uscente è arrivato con un lavoro non facile per i noti contrasti fra diverse 'scuole di pensiero', che hanno agitato la nostra società: questo Congresso deve contribuire a far uscire la SIU da un momento davvero difficile dimostrando che il valore di una Società scientifica non dipende dal carisma dei suoi rappresentanti ma dall'impegno scientifico e professionale di tutti i propri Soci. Sarà cioè il confronto scientifico del Congresso la rampa di lancio della nuova SIU.

A dispetto tuttavia di tutte queste difficoltà l'organizzazione del Congresso procede spedita: sono pervenuti quasi 400 contributi scientifici fra i quali il COSC ha accuratamente selezionato 113 comunicazioni, 85 poster e 38 video da presentare e discutere nel corso delle 3 giornate congressuali. Le differenti sessioni non sono state articolate, come nei precedenti congressi, seguendo rigidamente le modalità di esposizione (comunicazioni, video, poster), ma si è cercato di costruire delle sessioni monotematiche allo scopo di consentire ai partecipanti di seguire più facilmente gli argomenti di loro interesse, indipendentemente da come fossero presentati ed evitando di dover correre da una parte all'altra con l'orologio in mano e col rischio di perdere la presentazione e la discussione di qualche lavoro. Questa differente impostazione ha anche lo scopo di valorizzare i poster che sono un metodo di comunicazione rapido ed efficace e che non merita di essere ghettizzato nelle aule e negli orari di minor affluenza. Ci saranno anche relatori di altri Paesi Europei e degli USA in quanto il nostro Congresso Nazionale non può più essere il punto di incontro annuale di una comunità urologica chiusa in se stessa ma dovrà essere sempre più aperta al confronto con le realtà scientifiche degli altri Paesi.

I Main Topics sono rappresentati da una tavola rotonda sull'attualità e proiezione futura dell'oncologia urologica e da un Workshop sulla cistite recidivante nella donna con discussione interattiva. $\mathrm{Ci}$ sarà anche una seduta riservata al Club femminile di Urologia che è una giovane associazione delle donne urologo che merita di essere valorizzata non per i soliti motivi di pari opportunità (nella nostra specialità sono già ampiamente raggiunti), ma perché è formata da colleghe impegnate ed attive con rapporti e scambi culturali internazionali di grande valore.

E stato anche organizzato un Forum sui problemi giuridici ed assicurativi oggi così fortemente presenti nella nostra specialità chirurgica: i sentimenti della pubblica

\section{KARGER \\ Fax + 41613061234 \\ E-Mail karger@karger.ch} www. karger.com (c) 2000 S. Karger AG, Basel

Accessible online at: www. karger.com/journals/uin 
opinione, la conseguente interpretazione della legislazione vigente, le ricadute assicurative, lo stato d'animo dei professionisti. Gli interventi che si succederanno mostreranno, dai differenti punti di vista degli attori in scena, come e perché è cambiato il rapporto medico-paziente. Può essere che vengano esposte argomentazioni che siamo portati a rifiutare o che ci appaiono sgradevoli; al termine non è prevista alcuna discussione.

Da ultimo infine non possiamo e non vogliamo dimenticare la ricaduta economica che l'organizzazione del Congresso comporta per la nostra Società. La richiesta di partecipazione da parte dell'industria è stata massiccia e addirittura superiore alle aspettative: non è stato possibile accontentare tutti perché gli spazi a disposizione si sono a un certo punto esauriti. Tutto ciò testimonia quali e quanti interessi sia in grado di muovere la nostra Società. Possiamo pertanto già dire che l'organizzazione del Congresso produrrà un attivo la cui entità non è ovviamente ancora quantificabile. Sicuramente se i problemi che fisiologicamente sorgono all'interno della Società non venissero talora radicalizzati in 'guerre di religione' le spese di gestione verrebbero drasticamente ridotte sia pur con ram- marico dei molti consulenti (avvocati, commercialisti, notai) di volta in volta interpellati. La Società si è già adoperata per favorire un percorso di specializzazione per i giovani urologi con la creazione di borse di studio da utilizzarsi per stage in centri europei di riconosciuto valore. Tutti i Direttori dei centri in oggetto interpellati sulla disponibilità si sono dichiarati onorati ed hanno accettato senza riserve. La valorizzazione dei giovani urologi, la possibilità di aiutarli ad ampliare le conoscenze sui vari aspetti della specialità, l'opportunità di favorire la loro integrazione nell'ambito della comunità scientifica europea ed internazionale rappresentano obbiettivi che andrebbero perseguiti con determinazione. È fuor di dubbio che una maggior disponibilità economica di quella pur discreta attuale favorirebbe questo progetto. È persino inutile ricordare come una diminuzione dei contrasti riducendo le spese comporterebbe una maggiore disponibilità a iniziative come sopra esposto.

Prof. Alberto Reggiani Presidente del Congresso Dott. Claudio Ferri Segretario SIU 